\title{
Repeated implementation with overlapping generations of agents
}

\author{
Helmuts Āzacis ${ }^{1} \mathbb{D}$
}

Received: 2 August 2019 / Accepted: 28 January 2020 / Published online: 7 February 2020

(c) The Author(s) 2020

\begin{abstract}
We study repeated implementation in a model with overlapping generations of agents. A social choice function selects an alternative in each period as a function of preferences of those agents who are alive in that period. When the agents' preferences do not change during their lifetime, we show that any social choice function satisfying a mild unanimity condition is repeatedly implementable in subgame perfect equilibrium if there are at least three agents and they live sufficiently long. When the agents' preferences change every period, we show that only efficient social choice functions can be repeatedly implementable if the agents live sufficiently long.
\end{abstract}

\section{Introduction}

Implementation theory studies what goals a social designer can achieve when agents possess more information than the designer and they try strategically to benefit from it. So far, the literature has mostly focused on one-shot implementation, while the existing work on repeated implementation has assumed that the same agents are alive in all periods. However, the current debate about environmental protection, sustainable development or pension reform often involves arguments about intergenerational equity. To study whether intergenerational equity can be achieved, we need a setup that explicitly allows for different generations of agents.

I would like to thank the editor, the referee, Péter Vida as well as the participants of the 2018 Conference on Mechanism and Institution Design, the 2018 York Annual Symposium on Game Theory, the 4th Lancaster Game Theory Conference, and the 14th Meeting of the Society for Social Choice and Welfare for their comments that have helped to improve this paper.

Helmuts Āzacis

azacish@cf.ac.uk

1 Cardiff Business School, Cardiff University, Cardiff, UK 
Or said differently, once we introduce different generations, it gives rise to novel normative issues ${ }^{1}$ and we may want to know if these norms can be achieved when agents behave strategically. Therefore, our goal is to study what can or cannot be implemented in a setup with different generations of agents.

We now outline the model. Every period there are $n$ agents alive and these agents belong to different generations, that is, they have different ages. Every $T$ periods the oldest agent dies and a new agent is born. Thus, each agent lives exactly for $n T$ periods. Agents' preferences are additively separable over time and the current period preferences of agent depend on his type. The objective of designer is captured by a social choice function (SCF), which specifies a socially desirable alternative for every period as a function of types of those agents who are alive in that period. Because the designer never observes agents' types, she needs to design a sequence of mechanisms, called a regime, that allows her to elicit this information and, at the same time, to select the desired alternative in every period on the equilibrium path. If there exists such a regime, we say that the SCF is repeatedly implementable. Our goal is to characterize the SCFs that are repeatedly implementable. We assume complete information among agents and use subgame perfect equilibrium (SPE) as our solution concept.

We model generations similar to how it is done in the literature on repeated games played by overlapping generations (OLG) of players. For example, Cremer (1986) studies the possibilities of cooperation among players in such games when $T=1$, while Salant (1991), Kandori (1992) and Smith (1992) derive folk theorems by allowing $T$ to be large. The only difference with the current model is that in these papers, it is assumed that when an agent dies, he is replaced with his exact copy.

The OLG setup can be applied in many contexts. As mentioned, we can study whether intergenerational equity is achievable in the context of sustainable development or pension reform. Cremer (1986) and Kandori (1992) argue that the OLG games provide a natural theory of organizations. For example, different workers join and leave a firm at different times, which helps to sustain cooperation among them. From the perspective of implementation theory, we can ask what goals a manager can achieve if she is less informed than the workers. The US Senate is another example of institution that neatly fits the OLG framework: senators serve a six years' term and every two years, one third of them is reelected. Alternatively, we can think that there are $n$ different institutions (firms, countries) that are run by individuals (managers, heads of state) and, more often than not, individuals of different institutions are replaced at different times.

We study repeated implementation of SCFs under two opposite assumptions about the agents' types: when the agents' types do not change during their lifetime and when new types are drawn every period. Under the former assumption, we obtain the following results. We first show that any SCF that is repeatedly implementable, must satisfy a mild condition, which we call OLG-unanimity. It states that if all agents, no matter what their types are, agree on the best alternative and

\footnotetext{
1 For example, Shinotsuka et al. (2007) formulate three different notions of envy-freeness in a model with overlapping generations: No-Envy in Overlapping Consumptions, No-Envy in Lifetime Consumptions, and Equity in Lifetime Rate of Return. The first two notions would coincide in a static setup, while the last notion does not have an analogue in a static setup.
} 
if by lying, they can obtain this alternative in every period, then the SCF must be a constant function that always selects this alternative. Intuitively, it is impossible to incentivize the agents to reveal their true types because they already get their highest possible lifetime payoff.

Next, we construct a regime and show that any SCF that satisfies OLG-unanimity, is repeatedly implementable if there are at least three agents, $n \geq 3$, and they live long enough, namely, $T \geq 3$. Because agents' types do not change during their lifetime, a lie about their types does not need to be challenged immediately, but it can also be done by next generations. Also, OLG-unanimity condition implies that if an SCF is not a constant function, ${ }^{2}$ then there exists an agent who does not expect his highest possible lifetime payoff. This agent can always be incentivized to challenge the lie of other agents. This allows us to obtain very permissible results in terms of SCFs that are implementable. The regime that is used to prove the sufficiency result, borrows elements of the canonical mechanism that is used in one-shot implementation in SPE (Moore and Repullo 1988; Abreu and Sen 1990; Vartiainen 2007). We will elaborate on the connection to one-shot implementation in SPE in Sect. 3.1.

The results are different when the agents' types are drawn anew every period. We show now that if an SCF is not generation-efficient in the range, then it is not repeatedly implementable when $T$ is large. Generation-efficiency in the range extends the concept of efficiency in the range that was introduced by Lee and Sabourian (2011), to the OLG setup. It requires that there does not exist an SCF whose range is contained in the range of the SCF that is being implemented, but which gives higher expected payoff to every agent. The intuition for the result is as follows. If an SCF is not generation-efficient in the range, then by lying, the agents can obtain higher payoffs. Also, because types now change every period, any lie must be challenged and tested immediately. This, however, imposes a limit on the size of reward that the agent who challenges, can receive (otherwise he will also challenge when the others are not lying). Finally, for large enough $T$, the gains from sticking to the lie will exceed this reward. To show this result, we build on the aforementioned papers that prove folk theorems for OLG games and, specifically, on Smith (1992).

Repeated implementation has been studied by Kalai and Ledyard (1998), Chambers (2004), Lee and Sabourian (2011), Mezzetti and Renou (2017) and Azacis and Vida (2019). ${ }^{3}$ However, in all these papers, the same set of agents is alive in all periods. Thus, these papers do not consider the implementation of SCFs that can capture any intergenerational social choice considerations. Further, Kalai and Ledyard (1998) and Chambers (2004) assume that the state of the world is drawn only once and is kept fixed for all periods. Hence, their setup corresponds to the case in our model when the type of agent is kept fixed throughout his lifetime. Like us, they obtain very permissible results. On the other hand, Lee and Sabourian (2011); Mezzetti and Renou (2017); Azacis and Vida (2019) assume that in a model with

\footnotetext{
2 A constant SCF is trivially implementable.

3 Hayashi and Lombardi (2019) also study implementation in a dynamic setup, but in any given period, the socially desirable alternative depends not only on the state, but also on the history of alternatives that have been selected in the previous periods.
} 
discounting, a new state is drawn in each period. While the last two papers study what can be implemented for a given discount factor, Lee and Sabourian (2011) show that only SCFs that are efficient in the range, can be repeatedly implementable when the discount factor is sufficiently high. Thus, we provide a counterpart to their result in the OLG setup.

The rest of the paper is organized as follows. Section 2 formally describes the model. Section 3 deals with the case when the agents' types do not change during their lifetime, while Sect. 4 deals with the case when new types are drawn every period. Appendix A contains all the proofs.

\section{The model}

We consider a setup with overlapping generations of agents. Each generation consists of a single agent. Each agent lives for exactly $n T$ periods where $n$ and $T$ are two positive integers. Let $\mathbb{Z}$ be the set of integers equal or greater than $-n+1$. The agent of generation $z \in \mathbb{Z}$, or agent $z$ for short, is born at the beginning of period $z T$ and dies at the end of period $(z+n) T-1$. Hence, there are exactly $n$ agents alive in any period.

Let $A$ and $\Theta$ be, respectively, a set of feasible alternatives and a finite set of possible agent's types. Both sets remain the same over time. We assume that types are drawn independently and identically across generations according to a probability distribution $p$ such that $p(\theta)>0$ for each $\theta \in \Theta$. When types are assumed to be persistent, every agent's type is drawn only once and remains the same throughout his life. When types are assumed to be non-persistent, each period a new type for every agent who is alive, is drawn independently and identically also across time according to $p$. The payoff of agent $z$ in period $t=z T, \ldots,(z+n) T-1$ is $u\left(a_{t}, \theta_{z}^{t}\right)$ if the agent's type is $\theta_{z}^{t} \in \Theta$ and alternative $a_{t} \in A$ is implemented in that period, ${ }^{4}$ and his lifetime payoff is simply

$$
\sum_{t=z T}^{(z+n) T-1} u\left(a_{t}, \theta_{z}^{t}\right)
$$

We make the following assumptions about $u$. First, we assume that the agents have strict preferences over the alternatives for every realization of types:

Assumption A1 $u(a, \theta) \neq u(b, \theta)$ for all $a, b \in A$ such that $a \neq b$, and for all $\theta \in \Theta$.

Second, we assume that the change in the state leads to the change in the ordinal preferences:

\footnotetext{
${ }^{4}$ At the cost of additional notation, we could also allow $u$ to depend on the agent's identity, that is, to depend on $z$.
} 
Assumption A2 For every $\theta, \phi \in \Theta$ such that $\theta \neq \phi$ there exists a pair $a, b \in A$ such that $u(a, \theta)>u(b, \theta)$ and $u(a, \phi)<u(b, \phi)$.

Later it will be convenient to write the pair $(a, b)$ as $(a(\theta, \phi), b(\theta, \phi))$ with the convention that $a(\theta, \phi)$ is more desirable in state $\theta$ and $b(\theta, \phi)$ is more desirable in state $\phi$. Finally, we assume that the payoffs are bounded: $\inf _{(a, \theta) \in A \times \Theta} u(a, \theta)$ and $\sup _{(a, \theta) \in A \times \Theta} u(a, \theta)$ are finite.

We consider the implementation of socially desirable alternatives from period 0 onwards. In period $t$, a social choice function $f$ assigns an alternative in $A$ as a function of types of all agents who are alive in that period, that is, $f\left(\theta_{z-n+1}^{t}, \ldots, \theta_{z}^{t}\right) \in A$ where $z=\lfloor t / T\rfloor$ is the integer part when dividing $t$ by $T$. Note that the first argument of $f$ denotes the type of the oldest agent who is alive in that period; the second argument denotes the type of the second oldest agent who is alive and so on. Also note that $f$ is assumed to be time independent. That is, $f\left(\theta_{z-n+1}^{t}, \ldots, \theta_{z}^{t}\right)=f\left(\theta_{k-n+1}^{\tau}, \ldots, \theta_{k}^{\tau}\right)$ if $\left(\theta_{z-n+1}^{t}, \ldots, \theta_{z}^{t}\right)=\left(\theta_{k-n+1}^{\tau}, \ldots, \theta_{k}^{\tau}\right)$ for any $z$ and $k$ and any $t$ and $\tau$. Therefore, to describe $f$, it is enough to specify how $f$ depends on the types of agents $1, \ldots, n$.

A mechanism consists of messages that the agents can announce and an outcome function that selects a feasible alternative as a function of these messages. Let the message space of every agent in every period be $M$. It is without loss of generality, since we can always choose $M$ to be sufficiently large. Hence, we can associate every mechanism with its outcome function. We restrict attention to deterministic mechanisms in which the agents announce their messages simultaneously. Let $G$ be the set of all feasible mechanisms or, equivalently, outcome functions with a typical element $g$. Thus, given $g \in G$ and $m \in M^{n}$, alternative $a=g(m) \in A$ is implemented.

To avoid overcomplicating the notation, we next define histories and other relevant concepts for the case when types are persistent, which is the first case we analyse. When we will study the case with non-persistent types, we will clarify the necessary changes in the notation.

Since we now assume that agent's type remains the same during his lifetime, we write $\theta_{z}$ instead of $\theta_{z}^{t}$. A history of types at the start of period $t \geq 0$ is $\zeta_{t}=\left(\theta_{-n+1}, \ldots, \theta_{0}, \ldots, \theta_{\lfloor t / T]}\right)$, while a history of messages at the start of period $t>0$ is $\mu_{t}=\left(m^{0}, \ldots, m^{t-1}\right)$ where $m^{t}=\left(m_{\lfloor t / T\rfloor-n+1}^{t}, \ldots, m_{\lfloor t / T\rfloor}^{t}\right)$ is a profile of period $t$ messages with $m_{z}^{t}$ being the message of agent $z$. A history is $h_{t}=\left(\zeta_{t}, \mu_{t}\right)$ for $t>0$ and $h_{0}=\zeta_{0}$. Let $H_{t}$ denote the space of all period $t$ histories. We assume that the agents who are alive in period $t$, can distinguish between any two period $t$ histories. That is, we are in a complete and perfect information environment. On the other hand, the social designer cannot distinguish between any two period $t$ histories $h_{t}=\left(\zeta_{t}, \mu_{t}\right)$ and $h_{t}^{\prime}=\left(\zeta_{t}^{\prime}, \mu_{t}^{\prime}\right)$ if $\mu_{t}=\mu_{t}^{\prime}$, and $\zeta_{t}$ and $\zeta_{t}^{\prime}$ share the same first $n-1$ elements $\theta_{-n+1}, \ldots, \theta_{-1}$. That is, we assume that the types of those agents who were born before period 0 are also known to the designer.

A regime, $r$, describes which mechanism is selected after each possible history: $g_{t}=r\left(h_{t}\right) \in G$ subject to the restriction that $r\left(h_{t}\right)=r\left(h_{t}^{\prime}\right)$ if the designer cannot 
distinguish between histories $h_{t}$ and $h_{t}^{\prime}$. Note that we restrict attention to deterministic regimes. Because of that and also because the mechanisms are deterministic, it is fine to omit from the description of history $h_{t}$ which mechanisms and alternatives have been selected in periods $0, \ldots, t-1$. We assume that the designer commits to a regime at the start of period 0 and that the agents know which regime the designer employs.

A pure strategy of agent $z, s_{z}$, maps histories into messages: $s_{z}\left(h_{t}\right) \in M$ for all $t=z T, \ldots,(z+n) T-1$ and $h_{t} \in H_{t}{ }^{5}$ Let $S_{z}$ be the space of agent $z$ 's strategies. Let $s$ be a profile of strategies, one strategy for each $z \in \mathbb{Z}$. Also, let $s\left(h_{t}\right)=\left(s_{\lfloor t / T\rfloor-n+1}\left(h_{t}\right), \ldots, s_{\lfloor t / T\rfloor}\left(h_{t}\right)\right)$. Given $h_{t}$ and $s$, let $q\left(h_{t} \mid h_{t}, s\right)=1$ and for any $\tau>t$, let

$$
q\left(h_{\tau} \mid h_{t}, s\right)= \begin{cases}q\left(h_{\tau-1} \mid h_{t}, s\right) & \text { if } \tau / T \notin \mathbb{Z}, \zeta_{\tau}=\zeta_{\tau-1}, \mu_{\tau}=\left(\mu_{\tau-1}, s\left(h_{\tau-1}\right)\right), \\ q\left(h_{\tau-1} \mid h_{t}, s\right) p(\theta) & \text { if } \tau / T \in \mathbb{Z}, \zeta_{\tau}=\left(\zeta_{\tau-1}, \theta\right), \mu_{\tau}=\left(\mu_{\tau-1}, s\left(h_{\tau-1}\right)\right), \\ 0 & \text { otherwise. }\end{cases}
$$

For any $z \in \mathbb{Z}$, any $t=z T, \ldots,(z+n) T-1$, and any $h_{t}$, the (expected) payoff of agent $z$ for the rest of his life is

$$
v_{z}\left(s \mid h_{t}, r\right)=\sum_{\tau=t}^{(z+n) T-1} \sum_{h_{\tau} \in H_{\tau}} q\left(h_{\tau} \mid h_{t}, s\right) u\left(g_{\tau}\left(s\left(h_{\tau}\right)\right), \theta_{z}\right),
$$

where $\theta_{z}$ is the $z+n$-th element of $\zeta_{t}$ (since the first element of $\zeta_{t}$ is $\theta_{-n+1}$ ) and $g_{\tau}=r\left(h_{\tau}\right)$.

A strategy profile $s$ is a subgame perfect equilibrium (SPE) of $r$ if for all $z \in \mathbb{Z}$, all $t=z T, \ldots,(z+n) T-1$, all $h_{t} \in H_{t}$, and all $s_{z}^{\prime} \in S_{z}$, it is true that $v_{z}\left(s \mid h_{t}, r\right) \geq v_{z}\left(\left(s_{z}^{\prime}, s_{-z}\right) \mid h_{t}, r\right)$. A regime $r$ repeatedly implements $f$ in SPE if the set of SPE is non-empty and for each SPE $s$, we have that $g_{t}\left(s\left(h_{t}\right)\right)=f\left(\theta_{\lfloor t / T\rfloor-n+1}, \ldots, \theta_{\lfloor t / T\rfloor}\right)$ for all $t$ and $h_{t}$ such that $q\left(h_{t} \mid h_{0}, s\right)>0$, where $\left(\theta_{\lfloor t / T\rfloor-n+1}, \ldots, \theta_{\lfloor t / T\rfloor}\right)$ are the last $n$ elements of $\zeta_{t} . f$ is repeatedly implementable in SPE if there exists $r$ that repeatedly implements it in SPE.

\section{Persistent types}

We start with an example that illustrates the results for the case when agents' types do not change during their lifetime.

Example 1 Let $n=3, T \geq 3, A=\{a, b, c, d\}, \Theta=\left\{\theta, \theta^{\prime}\right\}$ with $p(\theta)=\frac{1}{2}$. The perperiod payoffs are given in the following table:

\footnotetext{
${ }^{5}$ From now on, $z T$ should be understood as $\max \{z T, 0\}$.
} 


\begin{tabular}{lll}
\hline & $u(\cdot, \theta)$ & $u\left(\cdot, \theta^{\prime}\right)$ \\
\hline$a$ & 0 & 0 \\
$b$ & 1 & 2 \\
$c$ & 2 & 1 \\
$d$ & 3 & 3 \\
\hline
\end{tabular}

The results of this section will tell that

- Any $f$ that never selects the best alternative $d$, is repeatedly implementable.

- If $f$ selects $d$ for some but not all type profiles, then it might not be implementable. For example, let $f\left(\theta_{1}, \theta_{2}, \theta_{3}\right)=d$ when $\left(\theta_{1}, \theta_{2}, \theta_{3}\right)=\left(\theta, \theta^{\prime}, \theta\right)$ or $\left(\theta_{1}, \theta_{2}, \theta_{3}\right)=\left(\theta^{\prime}, \theta, \theta^{\prime}\right)$, and $f\left(\theta_{1}, \theta_{2}, \theta_{3}\right)=a$ otherwise. ${ }^{6}$ If agents, irrespective of their true types, behave as if the types of consecutive agents alternate between $\theta$ and $\theta^{\prime}$, they can secure $d$ in every period. Nobody has incentives to deviate and $f$ is not implementable.

- Even if $f$ selects $d$ only for some type profiles, it might still be implementable. For example, let $f\left(\theta_{1}, \theta_{2}, \theta_{3}\right)=d$ when $\left(\theta_{1}, \theta_{2}, \theta_{3}\right)=\left(\theta, \theta^{\prime}, \theta\right)$ and $f\left(\theta_{1}, \theta_{2}, \theta_{3}\right)=a$ otherwise. This $f$ is implementable because agents cannot secure $d$ in every period.

The example suggests that implementation of SCF will only fail if every agent can obtain his maximal lifetime payoff when misrepresenting their types. We now proceed to show it formally. Let $f(\Theta)=\left\{a \in A \mid \exists\left(\theta_{1}, \ldots, \theta_{n}\right) \in \Theta^{n}\right.$ s.t. $\left.f\left(\theta_{1}, \ldots, \theta_{n}\right)=a\right\}$ denote the range of $f$. Let $\bar{a}(\theta)=\arg \max _{a \in A} u(a, \theta)$ and $\underline{a}(\theta)=\arg \min _{a \in A} u(a, \theta)$, which are assumed to exist. ${ }^{7}$ Let $\mathbb{Z}_{+}$be the set of non-negative integers.

Condition C1 (OLG-unanimity) If there exist $a \in A$ and an infinite sequence of types $\theta_{0}, \theta_{1}, \ldots$ such that

1. $\bar{a}(\theta)=$ a for all $\theta \in \Theta$, and

2. $f\left(\theta_{z}, \ldots, \theta_{z+n-1}\right)=$ a for all $z \in \mathbb{Z}_{+}$,

then $f\left(\theta_{1}^{\prime}, \ldots, \theta_{n}^{\prime}\right)=$ a for all $\left(\theta_{1}^{\prime}, \ldots, \theta_{n}^{\prime}\right) \in \Theta^{n}$, that is, $f$ is constant .

Condition $\mathrm{C} 1$ is a mild unanimity condition, which we call OLG-unanimity. The first premise of the condition says that all types agree on the best alternative, while the second premise ensures that $a$ can be selected by $f$ in every period. In particular, there exists an infinite sequence of types such that if one evaluates $f$ for any $n$ adjacent types in this sequence, the selected alternative will be $a$. Further, the statement of

\footnotetext{
${ }^{6}$ Recall that because $f$ is time independent, it is enough to specify how $f$ depends on the types of agents $1, \ldots, n$.

7 If the maximum did not exist for some $\theta$, then there must be $a \in A \backslash f(\Theta)$ such that $u(a, \theta)>u(b, \theta)$ for all $b \in f(\Theta)$. Then, let $\bar{a}(\theta)=a$. This will not affect the necessary condition as its premises only apply when $\bar{a}(\theta) \in f(\Theta)$. $\underline{a}(\theta)$ can be defined similarly.
} 
the second premise can be strengthened by noting that this infinite sequence of types consists of repetitions of the same finite sequence. This follows from the fact that $\Theta$ is finite and $f$ only depends on the types of $n$ consecutive generations. Hence, there must exist $k$ and $z$ in $\mathbb{Z}_{+}$such that $\left(\theta_{k}, \ldots, \theta_{k+n-1}\right)=\left(\theta_{z}, \ldots, \theta_{z+n-1}\right)$. But then we can construct another sequence that consists of repetitions of $\theta_{k}, \ldots, \theta_{z-1}$.

We claim that if the above two premises hold, then there exists an equilibrium in which $a$ is selected in every period irrespective of the realized types. Hence, if $f$ is repeatedly implementable, then it must be a constant function that selects $a$ for all possible realizations of types. The intuition is simple: $a$ is the best alternative for all types and they can ensure that it is selected in every period by pretending to have types as defined by the sequence $\theta_{0}, \theta_{1}, \ldots$. Clearly, no agent will have incentives to deviate. Therefore, there exists an equilibrium in which $a$ is implemented in every period. Thus, $f$ must be constant. Formally, ${ }^{8}$

Proposition 1 If $f$ is repeatedly implementable in SPE, then it must satisfy OLG-unanimity.

OLG-unanimity is satisfied by most (interesting) SCFs. For example, an SCF that selects an efficient alternative in every period (even in static sense), trivially satisfies it. Next we establish that OLG-unanimity is not only necessary but also sufficient if there are at least three agents alive at any moment and they live long enough.

Theorem 1 Suppose $n \geq 3$ and $T \geq 3$. Iff satisfies OLG-unanimity, then it is repeatedly implementable in SPE.

To prove the theorem, we construct a regime and show that it implements $f$ in SPE whenever $n \geq 3, T \geq 3$, and $f$ satisfies OLG-unanimity. Although the description of the regime is rather involved, it shares similarities with the canonical mechanism that is used for one-shot implementation in SPE. ${ }^{9}$ In a nutshell, in period $z T$, agents are asked to report the types of the three youngest agents. That is, on the one hand, period $z T$ messages are used to elicit the type of the newborn agent $z$, but on the other hand, they also allow the agents to confess if they have misreported the types of agents $z-2$ and $z-1$ in periods $(z-2) T$ and $(z-1) T$. Further, agent $z-1$ serves the role of "whistle-blower" in period $z T$. If he blows the whistle, then periods $z T+1$ and $z T+2$ are used to determine whether agent $z-1$ or other agents are lying. Therefore, we need that $n \geq 3$ and $T \geq 3$. $^{10}$

\footnotetext{
8 All proofs appear in Appendix A.

9 See, for example, Appendix B in Abreu and Sen (1990) for the description of the latter.

10 Theorem 1 can be extended to $T=1,2$ if $n$ is increased appropriately. This, however, requires changes in the regime because it is written assuming that $T \geq 3$.
} 


\subsection{Discussion}

Comparison with one-shot subgame perfect implementation. Moore and Repullo (1988), Abreu and Sen (1990) and Vartiainen (2007) have studied what can be implemented in SPE using dynamic mechanisms, but unlike repeated implementation, an alternative is selected only once at the very end. Despite this difference, the above results can be related to one-shot implementation in SPE. Therefore, we now briefly sketch the problem of one-shot implementation in SPE, which we adapt to make it comparable to our model.

Thus, let the set of agents be $N=\{1, \ldots, n\}$, let the set of states be $\Theta^{n}$ with a typical element $\vec{\theta}=\left(\theta_{1}, \ldots, \theta_{n}\right)$, let the set of alternatives be $\mathcal{A}=A^{n}$ with a typical element $\vec{a}=\left(a_{1}, \ldots, a_{n}\right)$, and let the payoff function of agent $z \in N$ be $u\left(\vec{a}, \theta_{z}\right)=\sum_{t=1}^{z} u\left(a_{t}, \theta_{z}\right)$. The objective of the designer is to implement alternative $f(\vec{\theta}) \in \mathcal{A}$ when the state is $\vec{\theta}$. To do that, the designer employs a multi-stage mechanism.

Abreu and Sen (1990) show in their Theorem 1 that if $f$ is one-shot implementable in SPE, then it satisfies the following condition with respect to some $B \subseteq \mathcal{A}$. $^{11}$

Condition C2 For all $(\vec{\theta}, \vec{\phi}) \in \Theta^{n} \times \Theta^{n}$ such that $f(\vec{\theta}) \neq f(\vec{\phi})$, there exist a sequence of agents $z(1), \ldots, z(K) \in N$ and a sequence of alternatives $\vec{a}_{1}, \ldots, \vec{a}_{K+1} \in B$ with $\vec{a}_{1}=f(\vec{\theta})$ such that

1. $u\left(\vec{a}_{k}, \theta_{z(k)}\right) \geq u\left(\vec{a}_{k+1}, \theta_{z(k)}\right)$ for $k=1, \ldots, K$,

2. $u\left(\vec{a}_{K}, \phi_{z(K)}\right)<u\left(\vec{a}_{K+1}, \phi_{z(K)}\right)$,

3. $\vec{a}_{k} \neq \arg \max _{\vec{a} \in B} u\left(\vec{a}, \phi_{z(k)}\right)$ for $k=1, \ldots, K$.

We can interpret our results in terms of Condition C2. First, one can set $K=3$ in Condition $\mathrm{C} 2^{12}$ and choose as $z(3)$ an agent whose type differs in states $\vec{\theta}$ and $\vec{\phi}$. Assumption A2 guarantees that this agent has a preference reversal between some $\vec{a}_{3}$ and $\vec{a}_{4}$ that is required by $\mathrm{C} 2.1$ and C2.2. Second, we can choose as $z(1)$ any agent for whom $\vec{a}_{1}=f(\vec{\theta})$ does not result in his highest possible payoff in state $\vec{\phi}$ as it would violate $\mathrm{C} 2.3$. In the OLG setup, the existence of such an agent is guaranteed by Condition $\mathrm{C}$ 1. That is, if $f$ is not a constant function, then Condition $\mathrm{C} 1$ is satisfied only if its premises are empty, which then implies that there always exists an agent who does not expect his maximal lifetime payoff. Finally, say, if $z(1)=n-1$, then we set $z(2)=n$. In the OLG setup, agent $n-1$ will die before agent $n$. Therefore, it is always possible to find $\vec{a}_{2}$ such that $u\left(f(\vec{\theta}), \theta_{n-1}\right) \geq u\left(\vec{a}_{2}, \theta_{n-1}\right)$ but $\vec{a}_{2}$ is not the worst outcome for agent $n$ in state $\vec{\theta}$. Because of the latter, it is also always possible to find $\vec{a}_{3}$ and $\vec{a}_{4}$ that satisfy C2.1 and C.2.2. For example, if $f(\vec{\theta})=\left(a_{1}, \ldots, a_{n-1}, a_{n}\right)$,

\footnotetext{
11 Vartiainen (2007) identifies the necessary and sufficient condition for one-shot implementation in SPE. Here, we focus on the more intuitive part of that condition.

12 But one cannot set $K=1$, that is, Maskin monotonicity of $f$ is not guaranteed. The implication is that strictly less SCFs are repeatedly implementable if we adopt Nash equilibrium as a solution concept.
} 
then we can set $\vec{a}_{2}=\left(a_{1}, \ldots, a_{n-1}, \bar{a}\left(\theta_{n}\right)\right), \vec{a}_{3}=\left(a\left(\theta_{z(3)}, \phi_{z(3)}\right), a_{1}, \ldots, a_{n-1}\right)$, and $\vec{a}_{4}=\left(b\left(\theta_{z(3)}, \phi_{z(3)}\right), a_{1}, \ldots, a_{n-1}\right) .^{13}$

Relaxing Assumption A1. If we allow for weak preferences, the OLG-unanimity condition remains necessary in its current form as long as the best alternative $\bar{a}(\theta)$ is unique for all $\theta$. The proof of Theorem 1 also remains true if $\bar{a}(\theta)$ is unique for all $\theta$ and, additionally, there exist two alternatives $c, d \in A$ such that no type is indifferent between them.

Relaxing Assumption A2. If we allow $T$ to be sufficiently large, we can replace Assumption A2 with the following one:

Assumption A3 For every $\theta, \phi \in \Theta$, there do not exist $\alpha>0$ and $\beta$ such that $u(a, \theta)=\alpha u(a, \phi)+\beta$ for all $a \in A$.

Assumption A3 says that for every pair $\theta, \phi \in \Theta$, we can always find two lotteries over the alternatives in $A$ (where the probabilities are rational numbers) such that type $\theta$ prefers one lottery over the other, while it is opposite for type $\phi$. But with each lottery we can associate a (finite) sequence of alternatives where the frequency of each alternative in the sequence is equal to the probability that this alternative is chosen in the corresponding lottery. Then, we can replace alternatives $a(\theta, \phi)$ and $b(\theta, \phi)$ that we use in the proof of Theorem 1, with the constructed sequences.

(Re)starting implementation. It is assumed that the designer knows the types of agents $-n+1, \ldots,-1$. The following example shows that it might not be possible to implement an SCF (from period 0) if the designer does not know their types.

Example 2 Let $n=3, T \geq 3, A=\{a, b, c\}, \Theta=\left\{\theta, \theta^{\prime}, \theta^{\prime \prime}\right\}$ with $p(\theta)=p\left(\theta^{\prime}\right)=\frac{1}{3}$. The per-period payoffs are given in the following table:

\begin{tabular}{llll}
\hline & $u(\cdot, \theta)$ & $u\left(\cdot, \theta^{\prime}\right)$ & $u\left(\cdot, \theta^{\prime \prime}\right)$ \\
\hline$a$ & 0 & 1 & 2 \\
$b$ & 1 & 0 & 1 \\
$c$ & 2 & 2 & 0 \\
\hline
\end{tabular}

Let $f\left(\theta_{1}, \theta_{2}, \theta_{3}\right)=c$ if $\theta_{1}=\theta$ and $f\left(\theta_{1}, \theta_{2}, \theta_{3}\right)=a$ otherwise. $f$ satisfies OLG-unanimity because there is no alternative that is best for all types. Therefore, $f$ is implementable if the designer knows the types of agents -2 and -1 .

Suppose now that the designer does not know their types. Also, suppose the types of agents $-2,-1$, and 0 are $\theta^{\prime}, \theta$, and $\theta$, respectively. If $f$ is implemented, then alternative $a$ is selected during periods $0, \ldots, T-1$ and alternative $c$ during periods $T, \ldots, 3 T-1$. However, if agents $-2,-1$, and 0 behave as if they all were of type $\theta$, then they can obtain alternative $c$ during periods $0, \ldots, 3 T-1$. None of these agents can be incentivized to deviate from this deception since they are getting their best alternative for the rest of their lives. Agent 1 might have incentives to challenge the

$13 a(\theta, \phi)$ and $b(\theta, \phi)$ are defined after Assumption A2. 
lie, but by the time he is born, agent -2 has already passed away. Thus, we conclude that $f$ cannot be implemented from period 0 if the designer does not know the types of agents -2 and -1 .

However, the implementation is still possible starting with period $(n-1) T$, that is, once agents $-n+1, \ldots,-1$ have passed away. To see it, fix arbitrary types for these agents. We also slightly modify the regime in the proof of Theorem 1. As mentioned before, the regime allows agents in period $z T$ to confess that they have lied about the types of agents $z-2$ and $z-1$ previously. The regime also allows agent $z-1$ to challenge the types of agents $z-2, z-1, z$ that are reported by other agents in period $z T$. Because the types of agents -2 and -1 are arbitrarily fixed, we need to preclude that the agents confess or challenge their types. With this modification, it is still true that in any equilibrium, the types of agents $0,1, \ldots$ are reported truthfully and, hence, $f$ is implemented from period $(n-1) T$ onwards.

The regime also has the property that once a deviation from the equilibrium play has occurred, a predetermined, infinite sequence of alternatives is implemented. This can be costly from the perspective of the designer. However, similar to how it is done in the previous paragraph, we can restart the implementation once the agents who are alive at the time of deviation, have passed away. Thus, the regime can be made robust to mistakes of agents.

Less than perfect information. We can relax the assumption that the agents who are alive in period $t$, can distinguish between any period $t$ histories. It is enough if the type of agent $z$ is common knowledge only among the agents who are alive in periods $z T,(z+1) T,(z+2) T$ as these are the periods when his type is elicited by the regime in the proof of Theorem 1 . Further, it is enough if only period $z T$ and $z T+1$ messages are observable for all $z \in \mathbb{Z}_{+}$and only by the agents who are alive in period $z T$. With such less than perfect information, Theorem 1 remains true if instead of SPE, we use extended subgame perfect equilibrium as the solution concept (for the definition, see page 877 in Kreps and Wilson 1982). ${ }^{14}$

\section{Non-persistent types}

Now we consider the other extreme when for every agent, a new type is drawn every period during his lifetime. Specifically, we assume that the types are drawn independently and identically both across periods and agents according to $p$. Also, we maintain Assumption A1, while Assumption A2 is not needed for the results of this section.

Lee and Sabourian (2011) consider a setup with infinitely-lived agents and payoff discounting and show in their Theorem 1 that when new types are drawn every period, any SCF that is not weakly efficient in the range cannot be repeatedly

\footnotetext{
14 It should also be noted that we do not use $p$ anywhere in the proof of Theorem 1. It is enough to assume that it is common knowledge among agents that every $\theta \in \Theta$ is realized with a strictly positive probability (which we use in the proof of Claim 1).
} 
implemented if the agents are sufficiently patient. We establish a similar negative result in the OLG setup when agents are sufficiently long-lived.

To show this result, we modify some of the notation and introduce some new one. Since now past realizations of types do not tell anything about the current period's types, it is convenient to denote the oldest agent in the current period as agent 1 , the second oldest agent as agent 2 , and so on up to the youngest agent who is denoted as agent $n$. Let $N=\{1, \ldots, n\}$. If $f$ is implemented, then the ex ante (that is, before the agent knows his type) per-period payoff of agent $i \in N$ is ${ }^{15}$

$$
u_{i}(f)=\sum_{\left(\theta_{1}, \ldots, \theta_{n}\right) \in \Theta^{n}} \prod_{j=1}^{n} p\left(\theta_{j}\right) u\left(f\left(\theta_{1}, \ldots, \theta_{n}\right), \theta_{i}\right) .
$$

Note that every agent's ex ante lifetime payoff is $T \cdot \sum_{i=1}^{n} u_{i}(f)$.

Let $\delta: \Theta^{n} \rightarrow \Theta^{n}$ be a deterministic deception where $\delta\left(\theta_{1}, \ldots, \theta_{n}\right)=\left(\theta_{1}^{\prime}, \ldots, \theta_{n}^{\prime}\right)$ says that the agents act as if their types were $\left(\theta_{1}^{\prime}, \ldots, \theta_{n}^{\prime}\right)$, although their true types are $\left(\theta_{1}, \ldots, \theta_{n}\right)$. Let the space of all deterministic deceptions be $D$. Also, let $f \circ \delta$ stand for the composite function of $f$ and $\delta$ : $f \circ \delta\left(\theta_{1}, \ldots, \theta_{n}\right)=f\left(\delta\left(\theta_{1}, \ldots, \theta_{n}\right)\right)$ for all $\left(\theta_{1}, \ldots, \theta_{n}\right) \in \Theta^{n}$. Finally, let $\tilde{\delta}$ be a random deception that selects a deterministic deception $\delta$ with probability $\tilde{\delta}(\delta)$ and let $\Delta$ be the space of all random deceptions. The payoff of agent $i \in N$ from a deception $\tilde{\delta}$ is $\sum_{\delta \in D} \tilde{\delta}(\delta) u_{i}(f \circ \delta)$, which we denote as $u_{i}(f \circ \tilde{\delta})$.

Definition 1 A social choice function $f$ is weakly efficient in the range if there does not exist $\tilde{\delta} \in \Delta$ such that $u_{i}(f \circ \tilde{\delta})>u_{i}(f)$ for all $i \in N$.

This is essentially the definition that Lee and Sabourian (2011) use, except that they convexify the set of payoffs without public randomization. We are, however, going to use a different definition of efficiency. In the setup of Lee and Sabourian (2011), there is no difference between the ex ante per-period payoffs and the average expected lifetime payoffs. This is not the case in the OLG setup. For example, the current second-oldest agent will become the oldest agent once the current oldest agent dies. The right notion of efficiency should take into account the lifetime payoffs. Therefore, we will use the following stronger notion of efficiency (see more discussion after Theorem 2):

Definition 2 A social choice function $f$ is weakly generation-efficient in the range if there does not exist $\tilde{\delta} \in \Delta$ such that $\sum_{j=1}^{i} u_{j}(f \circ \tilde{\delta})>\sum_{j=1}^{i} u_{j}(f)$ for all $i \in N$.

To simplify the analysis, we also make the following assumption about SCFs:

Assumption A4 There exist types $\theta_{1},\left(\theta_{2}, \ldots, \theta_{n}\right)$, and $\left(\theta_{2}^{\prime}, \ldots, \theta_{n}^{\prime}\right)$ such that $f\left(\theta_{1}, \theta_{2}, \ldots, \theta_{n}\right) \neq f\left(\theta_{1}, \theta_{2}^{\prime}, \ldots, \theta_{n}^{\prime}\right)$.

15 Thus, the subscript in $u_{i}(f)$ indicates the agent's age cohort. 
This assumption rules out SCFs that only vary with the type of the oldest agent. Unlike the setup of Lee and Sabourian (2011), agents live for a finite number of periods in our setup and the oldest agent might not have incentives to go along with a deception in his final periods. However, when $f$ satisfies Assumption $\mathrm{A} 4$, such incentives can be provided. For example, if $u\left(f\left(\theta_{1}, \theta_{2}^{\prime}, \ldots, \theta_{n}^{\prime}\right), \theta_{1}\right)>u\left(f\left(\theta_{1}, \theta_{2}, \ldots, \theta_{n}\right), \theta_{1}\right)$, then in state $\left(\theta_{1}, \theta_{2}, \ldots, \theta_{n}\right)$, the agents can behave as if the state was $\left(\theta_{1}, \theta_{2}^{\prime}, \ldots, \theta_{n}^{\prime}\right)$.

Theorem 2 Suppose $f$ satisfies Assumption $A 4$ and $n \geq 2$. If $f$ is not weakly generation-efficient in the range, then there exists $T^{*}$ such that for all $T \geq T^{*}, f$ is not repeatedly implementable in SPE.

Example 1 continued Let $f\left(\theta_{1}, \theta_{2}, \theta_{3}\right)=b$ when $\left(\theta_{1}, \theta_{2}, \theta_{3}\right)=\left(\theta^{\prime}, \theta^{\prime}, \theta^{\prime}\right)$ and $f\left(\theta_{1}, \theta_{2}, \theta_{3}\right)=c$ otherwise. This $f$ satisfies Assumption A4 because $f\left(\theta^{\prime}, \theta^{\prime}, \theta^{\prime}\right)=b \neq f\left(\theta^{\prime}, \theta, \theta\right)=c$. Also, $f$ is not weakly (generation) efficient. To see it, let $\delta\left(\theta^{\prime}, \theta^{\prime}, \theta\right)=\delta\left(\theta^{\prime}, \theta, \theta^{\prime}\right)=\delta\left(\theta, \theta^{\prime}, \theta^{\prime}\right)=\left(\theta^{\prime}, \theta^{\prime}, \theta^{\prime}\right)$ and $\delta\left(\theta_{1}, \theta_{2}, \theta_{3}\right)=\left(\theta_{1}, \theta_{2}, \theta_{3}\right)$ for all other configurations of types. With this deception, the alternative that is preferred by the majority of agents is selected. Then, $u_{i}(f \circ \delta)=\frac{14}{8}>u_{i}(f)=\frac{13}{8}$ for all $i=1,2,3$. The theorem says that $f$ is not repeatedly implementable if $T$ is large enough. On the other hand, $f$ satisfies Maskin monotonicity ${ }^{16}$ and, hence, is one-shot implementable in Nash equilibrium.

To prove the theorem, we borrow ideas from the literature on folk theorems for OLG games and, in particular, from Smith (1992). If $f$ is not weakly generation-efficient in the range, then there exists a deception $\delta \in \Delta$ such that $\sum_{j=1}^{i} u_{j}(f \circ \delta)>\sum_{j=1}^{i} u_{j}(f)$ for all $i \in N$. Because of Assumption A4, there also exists a deception $\gamma$ such that $u\left(f\left(\gamma\left(\theta_{1}, \theta_{2}, \ldots, \theta_{n}\right)\right), \theta_{1}\right) \geq u\left(f\left(\theta_{1}, \theta_{2}, \ldots, \theta_{n}\right), \theta_{1}\right)$ for all $\left(\theta_{1}, \theta_{2}, \ldots, \theta_{n}\right)$ with a strict inequality for at least one $\left(\theta_{1}, \theta_{2}, \ldots, \theta_{n}\right)$. Call the $T$ periods between the births of two consecutive agents as an overlap. We construct a strategy profile such that on the equilibrium path, agents deceive according to $\delta$ for the first $Q$ periods of each overlap and next they deceive according to $\gamma$ for the remaining $T-Q$ periods of every overlap. If a deviation from the path ever occurs, then the agents simply stop deceiving from that point on.

We prove that no agent has incentives to deviate from the equilibrium path for sufficiently large $T$, in three steps. First, the oldest agent does not have incentives to deviate during the last $T-Q$ periods of his life while he is being rewarded through the deception $\gamma$. Second, any gain that this agent can obtain from deviating in period $Q$, can be outweighed by the reward in the last $T-Q$ periods when $T-Q$ is sufficiently large. Thus, agent 1 does not want to deviate in period $Q$. The same is also true for the first $Q-1$ periods of the overlap because agents stop deceiving after any deviation and $u_{1}(f \circ \delta)>u_{1}(f)$. Third, we show that no agent $i=2, \ldots, n$ also

$\overline{{ }^{16} \text { Maskin monotonicity basically requires that } f \text { satisfies Condition } \mathrm{C} 2}$ for $K=1$. 
wants to deviate in any period of the overlap when $Q$ is sufficiently large because $\sum_{j=1}^{i} u_{j}(f \circ \delta)>\sum_{j=1}^{i} u_{j}(f)$ holds.

\subsection{Discussion}

Implementation in Nash equilibrium. Theorem 2 clearly remains valid if we replace SPE with Nash equilibrium as the solution concept. Note that Lee and Sabourian (2011) only prove their result for Nash equilibrium.

Weak efficiency in the range. If there exists $\delta \in \Delta$ such that $u_{i}(f \circ \delta)>u_{i}(f)$ for all $i \in N$, then it is also true that $\sum_{j=1}^{i} u_{j}(f \circ \delta)>\sum_{j=1}^{i} u_{j}(f)$ for all $i \in N$. That is, whenever $f$ is not weakly efficient in the range, then it is also not weakly generationefficient in the range. Therefore, if SCFs, which are not weakly generation-efficient in the range, cannot be repeatedly implemented, then it is also true about SCFs, which are not weakly efficient in the range. That is, the result that we prove is stronger than the one if we only assumed that $f$ is not weakly efficient in the range.

Relaxing Assumption A4. Although Assumption A4 can be relaxed, we claim that we cannot dispense of it completely. Thus, suppose that $f$ only varies with the type of agent 1 . Therefore, we now write $f\left(\theta_{1}\right)$ instead of $f\left(\theta_{1}, \ldots, \theta_{n}\right)$. Also, suppose that $f$ satisfies the following condition:

Condition C3 If $f\left(\theta_{1}\right) \neq f\left(\theta_{1}^{\prime}\right)$ for some $\theta_{1}, \theta_{1}^{\prime} \in \Theta$, then there exists $a \in A$ such that $u\left(f\left(\theta_{1}\right), \theta_{1}\right)>u\left(a, \theta_{1}\right)$ and $u\left(f\left(\theta_{1}\right), \theta_{1}^{\prime}\right)<u\left(a, \theta_{1}^{\prime}\right)$.

It is simply the definition of Maskin monotonicity, given that only agent 1 can serve as a whistle-blower.

We now argue that whenever $f$ satisfies Condition $\mathrm{C} 3$, one can design a regime such that no deception that results in an undesirable outcome, can be supported in equilibrium even if $f$ is not weakly generation-efficient in the range and $T$ is large. ${ }^{17}$ Thus, suppose that the agents deceive according to some $\delta$ (where now $\delta: \Theta^{n} \rightarrow \Theta$ ) in period $(z+n) T-1$, which is the last period for agent $z$. But because $f$ satisfies Condition $\mathrm{C} 3$, we can incentivize agent $z$ to deviate. Thus, the agents cannot deceive in that period. Suppose now that the agents deceive according to some $\delta$ in period $(z+n) T-2$. The payoff of agent $z$ from the deception is $u\left(f(\delta(\vec{\theta})), \theta_{1}\right)+u_{1}(f)$ where $\vec{\theta}=\left(\theta_{1}, \ldots, \theta_{n}\right)$. If he deviates from the deception, his payoff is $u\left(a, \theta_{1}\right)+v$ where $v$ is his expected payoff in period $(z+n) T-1$. We can always pick $v=u_{1}(f)$ and, by Condition C3, find $a$ such that $u(a, \delta(\vec{\theta}))<u(f(\delta(\vec{\theta})), \delta(\vec{\theta}))$ and $u\left(a, \theta_{1}\right)>u\left(f(\delta(\vec{\theta})), \theta_{1}\right)$ so that the deviation is profitable. Thus, the agents cannot deceive in period $(z+n) T-2$ either. Working backwards, the same argument also applies to the periods $(z+n-1) T, \ldots,(z+n) T-3$. Also, since $z$ was arbitrary, it follows that the agents will never deceive.

\footnotetext{
17 Note, however, we do not claim that $f$ is necessarily repeatedly implementable since there can be equilibria in which the agents do not follow a common deception in some period.
} 
Example 3 Let $n \geq 2, T \geq 1, A=\{a, b, c, d\}, \Theta=\left\{\theta, \theta^{\prime}\right\}$ with $p(\theta)=\frac{1}{2}$. The perperiod payoffs are given in the following table:

\begin{tabular}{lll}
\hline & $u(\cdot, \theta)$ & $u\left(\cdot, \theta^{\prime}\right)$ \\
\hline$a$ & 4 & 6 \\
$b$ & 6 & 4 \\
$c$ & 0 & 8 \\
$d$ & 8 & 0 \\
\hline
\end{tabular}

$f$ only depends on the type of agent 1 as follows: $f(\theta)=a$ and $f\left(\theta^{\prime}\right)=b$. Then, $u_{1}(f)=4$ and $u_{i}(f)=5$ for $i=2, \ldots, n$. Let $\delta(\vec{\theta})=\theta^{\prime}$ if $\theta_{1}=\theta$ and $\delta(\vec{\theta})=\theta$ if $\theta_{1}=\theta^{\prime}$. Then, $u_{1}(f \circ \delta)=6$ and $u_{i}(f \circ \delta)=5$ for $i=2, \ldots, n$. Hence, $f$ is not weakly generation-efficient in the range. Condition $\mathrm{C} 3$ is satisfied: 1) $u(f(\theta), \theta)>u(c, \theta)$ and $u\left(f(\theta), \theta^{\prime}\right)<u\left(c, \theta^{\prime}\right)$; 2) $u\left(f\left(\theta^{\prime}\right), \theta^{\prime}\right)>u\left(d, \theta^{\prime}\right)$ and $u\left(f\left(\theta^{\prime}\right), \theta\right)<u(d, \theta)$. The discussion in the previous paragraph says that no deception (not just $\delta$ ) can be maintained in the equilibrium. ${ }^{18}$

Open Access This article is licensed under a Creative Commons Attribution 4.0 International License, which permits use, sharing, adaptation, distribution and reproduction in any medium or format, as long as you give appropriate credit to the original author(s) and the source, provide a link to the Creative Commons licence, and indicate if changes were made. The images or other third party material in this article are included in the article's Creative Commons licence, unless indicated otherwise in a credit line to the material. If material is not included in the article's Creative Commons licence and your intended use is not permitted by statutory regulation or exceeds the permitted use, you will need to obtain permission directly from the copyright holder. To view a copy of this licence, visit http://creativeco mmons.org/licenses/by/4.0/.

\section{A proofs}

Proof of Proposition 1 Suppose $f$ is repeatedly implementable in SPE. Let $s$ denote an SPE. Suppose that the premises of Condition C1 hold. Using $s$, we will construct another equilibrium strategy profile $s^{\prime}$ that implements alternative $a$ forever after some history where $a$ is defined in Condition $\mathrm{C} 1$.

Let $\theta_{-n+1}, \ldots, \theta_{-1}$ be given. Let $\theta_{0}, \theta_{1}, \ldots$ be the sequence defined in Condition $\mathrm{C} 1$. Let $\zeta_{t}=\left(\theta_{-n+1}, \ldots, \theta_{\lfloor t / T\rfloor}\right)$ be period $t$ history of types that is obtained from these two sequences. Let $\mu_{t}=\left(\mu_{t-1}, s\left(h_{t-1}\right)\right)$ be period $t$ history of messages and $h_{t}=\left(\zeta_{t}, \mu_{t}\right)$ be period $t$ history (which are obtained recursively) that would arise if the agents followed strategies $s$ and the history of types was $\zeta_{t}$. We reserve notation $\zeta_{t}, \mu_{t}, h_{t}$ to these particular histories. Also note that these histories occur with a strictly positive probability if the agents follow strategies $s$.

\footnotetext{
18 Even more, one can argue that this $f$ is repeatedly implementable using a regime, which is similar to the one in Mezzetti and Renou (2017) or Azacis and Vida (2019) if $n \geq 3$.
} 
For any period $t \geq n T$, let $\Theta_{t}$ denote the set of period $t$ histories of types that has $\zeta_{n T}$ as a sub-history and let $H_{t}^{\prime}=\left\{h_{t}^{\prime}=\left(\zeta_{t}^{\prime}, \mu_{t}\right) \mid \zeta_{t}^{\prime} \in \Theta_{t}\right\}$. Thus, $H_{t}^{\prime}$ consists of histories that only differ from $h_{t}$ in their history of types after period $n T$. Let $H_{t}^{\prime}=\left\{h_{t}\right\}$ for all $t=0, \ldots, n T-1$. Let $s^{\prime}$ be defined as follows: $s^{\prime}\left(h_{t}^{\prime}\right)=s\left(h_{t}\right)$ for all $h_{t}^{\prime} \in H_{t}^{\prime}$ and $s^{\prime}\left(h_{t}^{\prime \prime}\right)=s\left(h_{t}^{\prime \prime}\right)$ for all $h_{t}^{\prime \prime} \in H_{t} \backslash H_{t}^{\prime}$. Thus, after any $h_{t}^{\prime} \in H_{t}^{\prime}$, $s^{\prime}$ tells the agents to send the same messages as after history $h_{t}$.

If the agents follow strategies in $s^{\prime}$, alternative $a$ is implemented forever after history $h_{n T}$ irrespective of the realised types. We verify that $s^{\prime}$ is an SPE. Since $s$ is an SPE, then by definition, $s^{\prime}$ implies Nash equilibrium play in subgames starting after any $h_{t}^{\prime \prime} \in H_{t} \backslash H_{t}^{\prime}$. Consider a subgame after any $h_{t}^{\prime} \in H_{t}^{\prime}$ for $t \geq n T$. Since the agents receive their best alternative in every period, they do not have incentives to deviate. Finally, consider a subgame after $h_{t}$ for $t=0, \ldots, n T-1$. The payoff of any agent who is alive in period $t$ and deviates from $s^{\prime}$, is the same as his payoff if he deviated from $s$. On the other hand, his payoff if he does not deviate from $s^{\prime}$ is higher than his payoff if he follows $s$ because he obtains his best alternative in future periods with higher probability. Since he did not have incentives to deviate from $s$, he does not have incentives to deviate from $s^{\prime}$. Thus, $s^{\prime}$ is an SPE. But then, if $f$ is repeatedly implementable in SPE, it must be a constant function that always selects $a$.

Proof of Theorem 1 Any constant $f$ is trivially implementable. Therefore, we assume that $f$ is not constant. But then Condition $\mathrm{C} 1$ will only be satisfied if its premises are empty.

Let $\bar{b}(\theta)=\arg \max _{a \in f(\Theta) \backslash\{\bar{a}(\theta)\}} u(a, \theta)$ denote the best alternative for type $\theta$ in the range of $f$, which is different from $\bar{a}(\theta)$.

As part of the regime, we are going repeatedly to use the following game:

The integer game. Let $M=\Theta^{3} \times \mathbb{Z}_{+}$. Suppose we are in period $t$. Given period $t$ messages $m^{t} \in M^{n}$, let $k$ be the agent who has announced the highest integer and suppose $m_{k}^{t}=(\psi, \cdot, \cdot, \cdot)$. (Ties are always broken in favour of the agent with the highest index.) Let $z$ be the youngest agent in period $t$. Then,

1. If $k=z$, then $g\left(m^{t}\right)=\bar{b}(\psi)$ and $g\left(m^{\tau}\right)=\bar{a}(\psi)$ for all $\tau>t$ and all $m^{\tau}$.

2. If $k \neq z$, then $g\left(m^{t}\right)=g\left(m^{\tau}\right)=\bar{a}(\psi)$ for all $\tau=t+1, \ldots,(k+n) T-1$ and $g\left(m^{\tau}\right)=\bar{b}(\psi)$ for all $\tau>(k+n) T-1$ and all $m^{\tau}$.

It is easy to see that the integer game does not have an equilibrium, even if all agents have identical preferences: the youngest agent prefers if part 1 applies, while the second youngest agent prefers if part 2 applies. ${ }^{19}$ (Note that the assumptions that $\bar{a}(\psi)$ is unique, $n \geq 3$, and $T \geq 2$ play the role.)

The regime $r$ is defined as follows. Let $M=\Theta^{3} \times \mathbb{Z}_{+}$. We fix two distinct, arbitrary alternatives $c, d \in A$. For any period $t$, let $N_{t}=\{\lfloor t / T\rfloor-n+1, \ldots,\lfloor t / T\rfloor\}$

\footnotetext{
${ }^{19}$ In the regime below, the same result still holds even though the message space when the integer game will apply, will be a subset of $M^{n}$.
} 
denote the set of agents who are alive in that period. Let $\theta_{-n+1}, \ldots, \theta_{-1}$ be given. We apply the following algorithm starting with period $t=0$ :

0 . Let $z=t / T$.

1. If there is $\left(\eta_{z-2}, \eta_{z-1}, \eta_{z}\right) \in \Theta^{3}$ s.t. $m_{k}^{t}=\left(\eta_{z-2}, \eta_{z-1}, \eta_{z}, \cdot\right)$ for at least $n-1$ agents $k \in N_{t}$, then let $g\left(m^{t}\right)=f\left(\theta_{z-n+1}, \ldots, \theta_{z-1}, \eta_{z}\right)$.

(a) If $m_{z-1}^{t}=\left(\eta_{z-2}, \eta_{z-1}, \eta_{z}, \cdot\right)$ and $\left(\eta_{z-2}, \eta_{z-1}\right)=\left(\theta_{z-2}, \theta_{z-1}\right)$, then let $g\left(m^{\tau}\right)=f\left(\theta_{z-n+1}, \ldots, \theta_{z-1}, \eta_{z}\right)$ for all $\tau=t+1, \ldots, t+T-1$ and all $m^{\tau}$. (Thus, the messages in periods $\tau=t+1, \ldots, t+T-1$ play no role.) Set $\theta_{z}=\eta_{z}$ and $t=(z+1) T$, and go back to part 0 .

(b) If $m_{z-1}^{t}=\left(\eta_{z-2}, \eta_{z-1}, \eta_{z}, \cdot\right)$ but $\left(\eta_{z-2}, \eta_{z-1}\right) \neq\left(\theta_{z-2}, \theta_{z-1}\right)$, then $g\left(m^{\tau}\right)=c$ for $\tau=t+1, g\left(m^{\tau}\right)=d$ for $\tau=t+2, \ldots, t+T-1$, and $g\left(m^{\tau}\right)=c$ for $\tau \geq t+T$ and all $m^{\tau}$.

(c) If $m_{z-1}^{t}=\left(\phi_{z-2}, \phi_{z-1}, \phi_{z}, \cdot\right) \neq\left(\eta_{z-2}, \eta_{z-1}, \eta_{z}, \cdot\right)$, then let $i \in\{z-2, z-1, z\}$ s.t. $\phi_{i} \neq \eta_{i}$. Set $t=z T+1$ and go to part 3 .

2. For the messages that do not fall under part 1 , the outcome is determined by the integer game.

3. In period $t=z T+1$,

(a) If $m_{k}^{t}=(\cdot, \cdot, \cdot, 0)$ for at least $n-1$ agents $k \in N_{t}$, then $g\left(m^{t}\right) \stackrel{g}{=} g\left(m^{\tau}\right)=\bar{a}\left(\phi_{z-1}\right)$ for all $\tau=t+1, \ldots,(z+n-1) T-1$ and $g\left(m^{\tau}\right)=\bar{b}\left(\phi_{z-1}\right)$ for $\tau \geq(z+n-1) T$ and all $m^{\tau}$.

(b) If $m_{k}^{t}=(\cdot, \cdot, \cdot, 1)$ for at least $n-1$ agents $k \in N_{t}$, including agent $z$, then $g\left(m^{t}\right)=g\left(m^{\tau}\right)=\underline{a}\left(\eta_{z-1}\right)$ for all $\tau=t+1, \ldots,(z+n-1) T-1$, $g\left(m^{\tau}\right)=\bar{a}\left(\eta_{z-1}\right)$ for $\tau=(z+n-1) T,(z+n-1) T+1$, and $g\left(m^{\tau}\right)=\bar{a}\left(\psi_{z}\right)$ for all $\tau \geq(z+n-1) T+2$ and all $m^{\tau}$ where $\psi_{z}$ is given by $m_{z}^{t}=\left(\psi_{z}, \cdot, \cdot, 1\right)$.

(c) If $m_{k}^{t}=(\cdot, \cdot, \cdot, 1)$ for all $k \in N_{t} \backslash\{z\}$ while $m_{z}^{t}=\left(\psi_{z}, \cdot, \cdot, l\right)$ where $l \neq 1$, then $g\left(m^{t}\right)=\underline{a}\left(\eta_{z-1}\right)$. Set $t=z T+2$ and go to part 4 .

(d) For all other messages, the outcome is determined by the integer game.

4. In period $t=z T+2$,

(a) If $m_{k}^{t}=(\cdot, \cdot, \cdot, 0)$ for at least $n-1$ agents $k \in N_{t}$, then $g\left(m^{t}\right)=\bar{a}\left(\eta_{z-1}\right)$ and $g\left(m^{\tau}\right)=\bar{a}\left(\psi_{z}\right)$ for all $\tau>t$ and all $m^{\tau}$.

(b) If $m_{k}^{t}=(\cdot, \cdot, \cdot, 1)$ for at least $n-1$ agents $k \in N_{t}$, including agent $i$, then $g\left(m^{t}\right)=a\left(\eta_{i}, \phi_{i}\right), g\left(m^{t+1}\right)=g\left(m^{t+2}\right)=\bar{a}\left(\eta_{z-1}\right)$, and $g\left(m^{\tau}\right)=\underline{a}\left(\eta_{z-1}\right)$ for all $\tau>t+2$ and all $m^{\tau}$.

(c) If $m_{k}^{t}=(\cdot, \cdot, \cdot, 1)$ for all $k \in N_{t} \backslash\{i\}$ while $m_{i}^{t}=(\cdot, \cdot, \cdot, l)$ where $l \neq 1$, then $g\left(m^{t}\right)=b\left(\eta_{i}, \phi_{i}\right), g\left(m^{t+1}\right)=g\left(m^{t+2}\right)=\bar{a}\left(\eta_{z-1}\right)$, and $g\left(m^{\tau}\right)=\underline{a}\left(\eta_{z-1}\right)$ for all $\tau>t+2$ and all $m^{\tau}$.

(d) For all other messages, the outcome is determined by the integer game. 
If period $z T$ messages fall under part 1a, we will say that they are unanimous. Note that if the messages are unanimous in period $z T$, then they must also have been unanimous in periods $k T$ for all $0 \leq k<z$. We will say that the unanimous messages are truthful if the agents report their true types.

We first construct equilibrium strategies that implement $f$ in SPE. Let $\theta_{z}$ denote the true type of agent $z$ for all $z \in \mathbb{Z}$. In period $t=0$, let $m_{k}^{0}=\left(\theta_{-2}, \theta_{-1}, \theta_{0}, \cdot\right)$ for all $k \in N_{0}$. For any $t>0$, let $m_{k}^{t}=\left(\theta_{\lfloor t / T\rfloor-2}, \theta_{\lfloor t / T\rfloor-1}, \theta_{\lfloor t / T\rfloor}, \cdot\right)$ for all $k \in N_{t}$ if agents sent unanimous messages in period $\lfloor(t-1) / T\rfloor T$; otherwise, $m_{k}^{t}=\left(\theta_{k}, \cdot, \cdot, 1\right)$ for all $k \in N_{t}$. In words, the strategies require every period to announce the true types of the three youngest agents who are alive in that period as long as the last time when a new agent was born, the agents sent unanimous (either truthful or untruthful) messages; otherwise, keep announcing one's own true type and integer 1 forever. If they follow these strategies, $f$ is clearly implemented in every period. Agent $z-1$ can unilaterally change the outcome only if he deviates in period $z T$. Given the defined strategies, the deviation will trigger part $3 \mathrm{~b}$. Since the other agents have announced the true type of agent $z-1$, this agent will receive his worst alternative $\underline{a}\left(\theta_{z-1}\right)$ in every period for the rest of his life starting with period $z T+1$ and the deviation will not affect what he receives in period $z T$. Hence, the deviation is not profitable.

We also need to check if the defined strategies form Nash equilibria in the subgames that follow agent $z-1$ 's deviation in period $z T$. As mentioned, the deviation will trigger part $3 \mathrm{~b}$ in period $z T+1$. Only agent $z$ can unilaterally change this outcome. First, it is easy to verify that he cannot gain by misreporting his type, that is, it is always optimal to announce $\psi_{z}=\theta_{z}$ once parts 3 and 4 of the regime are triggered. (Note that whenever $\psi_{z}$ matters for the selection of alternative, the selected alternative is $\bar{a}\left(\psi_{z}\right)$.) Second, by announcing a different integer, he can trigger part $3 c$, in which case, given others' strategies, he expects the outcome corresponding to part $4 \mathrm{~b}$ in the next period. Again, it is easy to verify that agent $z$ does not gain from such a deviation.

Also, we can check that no profitable deviation exists from part $4 \mathrm{~b}$. Only agent $i$ can unilaterally change the outcome and only to part 4c. Since his true type was announced, $\eta_{i}=\theta_{i}$, in period $z T$, he prefers $a\left(\eta_{i}, \phi_{i}\right)$ to $b\left(\eta_{i}, \phi_{i}\right)$ for any $\phi_{i} \neq \eta_{i}$. Therefore, the deviation to part $4 \mathrm{c}$ is not profitable. Finally, no profitable deviation exists after any other history simply because the outcome of the regime does not depend on the messages that are sent after these other histories.

In the remainder of the proof, we will argue that there do not exist undesirable equilibria. Suppose that in an equilibrium, the agents have been sending unanimous, but possibly untruthful messages up to some period $z T$. Also suppose that period $z T$ messages fall under part $1 \mathrm{c}$. We will now analyse what are the equilibrium outcomes of the subgame that follows these messages. That is, suppose that period $t=z T$ messages are $m_{k}^{t}=\left(\eta_{z-2}, \eta_{z-1}, \eta_{z}, \cdot\right)$ for all $k \in N_{t} \backslash\{z-1\}$ while $m_{z-1}^{t}=\left(\phi_{z-2}, \phi_{z-1}, \phi_{z}, \cdot\right) \neq\left(\eta_{z-2}, \eta_{z-1}, \eta_{z}, \cdot\right)$. Assume first that $\phi_{z-2}, \phi_{z-1}, \phi_{z}$ are the true types of agents $z-2, z-1, z$.

Suppose part 4 is reached in period $z T+2$. There is an equilibrium that falls under part $4 \mathrm{a}$, in which everyone announces 0 . There cannot be an equilibrium falling under part $4 \mathrm{~b}$ : by assumption, $\phi_{i}$ is the true type of agent $i$. Therefore, he prefers $b\left(\eta_{i}, \phi_{i}\right)$ to $a\left(\eta_{i}, \phi_{i}\right)$ and will deviate to trigger part $4 \mathrm{c}$. Neither there can 
be an equilibrium falling under part $4 \mathrm{c}$ because $\bar{a}\left(\eta_{z-1}\right) \neq \underline{a}\left(\eta_{z-1}\right)$ and agent $k \in\{z-1, z\} \backslash\{i\}$ is better off by triggering part $4 \mathrm{~d}$. Finally, since the integer game does not have an equilibrium, there cannot be an equilibrium falling under part $4 \mathrm{~d}$. It follows that if part 4 is reached, the unique equilibrium outcome corresponds to part $4 \mathrm{a}$.

Consider now period $z T+1$ and part 3 of the regime. There is an equilibrium that falls under part $3 \mathrm{a}$, in which everyone announces 0 . There cannot be an equilibrium falling under part $3 \mathrm{~b}$ because agent $z$ is better off by announcing his true type, $\psi_{z}=\phi_{z}$, and triggering part $3 \mathrm{c}$ as he expects the outcome corresponding to part $4 \mathrm{a}$ in period $z T+2$. Neither there can be an equilibrium falling under part $3 \mathrm{c}$ because $\underline{a}\left(\eta_{z-1}\right) \neq \bar{a}\left(\eta_{z-1}\right)$ and agent $z-1$ would prefer to deviate and trigger part $3 \mathrm{~d}$. Finally, there cannot be an equilibrium falling under part $3 \mathrm{~d}$. To summarize, if other agents misreport the type of at least one of the agents $z-2, z-1, z$ in period $z T$, then agent $z-1$, by announcing the true types of agents $z-2, z-1, z$, can secure his most preferred outcome from period $z T+1$ onwards (without affecting the outcome of period $z T$ ).

Assume now that period $z T$ messages still fall under part $1 \mathrm{c}$, but agent $z-1$ does not announce the true type of at least one of the agents $z-2, z-1, z$. It is still true that in the subgame that follows, parts $3 \mathrm{~d}$ and $4 \mathrm{~d}$ cannot be played on the equilibrium path. Part $3 \mathrm{c}$ also cannot be played on the equilibrium path: if it is triggered, period $z T+2$ messages will fall under one of the parts $4 \mathrm{a}-4 \mathrm{c}$. But then, since $\bar{a}\left(\eta_{z-1}\right) \neq \underline{a}\left(\eta_{z-1}\right)$, agent $z-1$ does not obtain his best outcome starting period $z T+1$ and is better off by triggering part $3 \mathrm{~d}$. Thus, only parts $3 \mathrm{a}$ and $3 \mathrm{~b}$ can possibly be played in equilibrium after agent $z-1$ triggers $1 \mathrm{c}$.

Given the above conclusions, we now argue that the only possible equilibria are in unanimous messages, that is, only part $1 \mathrm{a}$ is played in any equilibrium. Clearly, period $z T$ messages cannot fall under part 2. Neither they can fall under part 1c: from the previous analysis, we know that either part $3 \mathrm{a}$ or $3 \mathrm{~b}$ will be played in the continuation. However, agent $z$ then is better off by triggering part 2. Suppose now that period $z T$ messages fall under part $1 \mathrm{~b}$. In this case, neither agent $z-2$, nor agent $z-1$ obtains his best alternative in every period. Therefore, depending whether their types are misreported in period $(z-1) T$ or in $z T$, either agent $z-2$ will deviate in period $(z-1) T$ or agent $z-1$ will deviate in period $z T$ to part $1 \mathrm{c}$ by announcing the true types and will expect part $3 \mathrm{a}$ to be played in the next period, giving him his most preferred outcome. ${ }^{20}$ Thus, part $1 \mathrm{~b}$ also cannot be played in equilibrium. It follows that the only equilibria are in unanimous messages.

It remains to argue that the unanimous messages must be truthful in any equilibrium. From the previous analysis, we know that agent $z$, by triggering part $1 \mathrm{c}$, can secure his most preferred outcome from period $(z+1) T+1$ onwards when others lie about the types of agents $z-1, z, z+1$ in period $(z+1) T$. We only need to show that in any potential equilibrium with unanimous, but untruthful messages, there always exists some agent $z$ who does not expect his maximal payoff from period

${ }^{20}$ This is the only instance in the proof where we need that agent $z-1 \mathrm{can}$ trigger part $1 \mathrm{c}$ by challenging the type of agent $z$. 
$(z+1) T+1$ onwards and, hence, has the incentives to deviate. Note that it is equivalent to saying that the agent does not expect his maximal payoff from period $(z+1) T$ on because the alternative that is specified by an SCF, does not change during periods $(z+1) T, \ldots,(z+2) T-1$. That there exists such an agent is proven in the following claim.

Claim 1 Suppose there exists an equilibrium in unanimous but untruthful messages. Then, with a strictly positive probability, there exists an agent, say, agent $z$ and a type of this agent, say, $\phi$ such that in the equilibrium, this agent expects less than his maximal possible payoff of $(n-1) T u(\bar{a}(\phi), \phi)$ from period $(z+1) T$ on and the agents misreport either the type of agent $z-1, z$, or $z+1$.

Proof of Claim 1 Consider an equilibrium in unanimous but untruthful messages.

Because the premises of Condition $\mathrm{C} 1$ are empty, either there is no $a \in A$ that is the best alternative for all types, or there is no sequence of types $\theta_{0}, \theta_{1}, \ldots$ such that $f\left(\theta_{z}, \ldots, \theta_{z+n-1}\right)=a$ for all $z \in \mathbb{Z}$. We will consider each of these two cases separately.

Suppose first there does not exist $a \in A$ that is the best alternative for all types. Suppose that the agents in period $z T$ lie about the true type, say, $\phi$ of agent $z$. Then, they must also lie about his type in periods $(z+1) T$ and $(z+2) T$. If this agent does not receive his maximal payoff from period $(z+1) T$ on, we are done with the claim. If he receives the maximal payoff, it must be that alternative $\bar{a}(\phi)$ is implemented in every period starting with period $(z+1) T$. But by the assumption, there exists a type of agent $z+1$ for whom $\bar{a}(\phi)$ is not the best alternative. Since $n \geq 3$, this agent does not get his maximal payoff from period $(z+2) T$ on, which again establishes the claim.

Suppose now that there exists $a \in A$ that is the best alternative for all types, but there does not exist an infinite sequence of types $\theta_{0}, \theta_{1}, \ldots$ such that $f\left(\theta_{z}, \ldots, \theta_{z+n-1}\right)=a$ for all $z \in \mathbb{Z}$. Let $\phi_{0}, \phi_{1}, \ldots$ denote the true types and $\theta_{0}, \theta_{1}, \ldots$ denote the reported types of agents $0,1, \ldots$. Suppose that $\theta_{z} \neq \phi_{z}$, but agent $z$ expects the maximal possible payoff from period $(z+1) T$ on, that is, alternative $a$ is implemented (at least) during periods $(z+1) T, \ldots,(z+n) T-1$. We claim that there exists a profile of types $\left(\phi_{z+1}, \ldots, \phi_{z+n-1}\right) \in \Theta^{n-1}$ such that the type of at least one of the agents among $z+1, \ldots, z+n-1$ is misreported. Suppose not. Then, it must be that $f\left(\theta_{z}, \phi_{z+1}, \ldots, \phi_{z+n-1}\right)=a$ for all $\left(\phi_{z+1}, \ldots, \phi_{z+n-1}\right) \in \Theta^{n-1}$. But this contradicts the assumption that there does not exist an infinite sequence of types $\theta_{0}, \theta_{1}, \ldots$ such that $f\left(\theta_{k}, \ldots, \theta_{k+n-1}\right)=a$ for all $k \in \mathbb{Z}$ : if every agent, irrespective of his true type, reported that his type is $\theta_{z}$, the alternative $a$ would be selected forever. Thus, there exists a realization of types $\phi_{z+1}, \ldots, \phi_{z+n-1}$ such that the type of some agent among $z+1, \ldots, z+n-1$ is misreported. Pick this agent (if there exist several, then pick the one with the highest index), and repeat now the above argument for this agent. Since we have assumed that there does not exist an infinite sequence of types $\theta_{0}, \theta_{1}, \ldots$ that would allow the agents to obtain alternative $a$ forever, ultimately there 
must be an agent $k$ whose type is misreported and who does not receive his highest possible payoff from period $(k+1) T$ on. This completes the proof of the claim.

To conclude, in any potential equilibrium with unanimous, but untruthful messages, there always exists an agent who has incentives to deviate. ${ }^{21}$ Hence, the only equilibria are in unanimous and truthful messages. Consequently, the desired alternatives will always be selected on the equilibrium path.

Proof of Theorem 2 Suppose there exists a regime that repeatedly implements $f$ in SPE. Let $s$ be an SPE. Given $s$, we are going to construct another strategy profile $s^{\prime}$ that also forms an SPE for sufficiently large $T$ and that results in undesirable outcomes.

We start by defining the deceptions that the agents will use. Since $f$ satisfies Assumption A4, there exist types $\hat{\theta}_{1},\left(\hat{\theta}_{2}, \ldots, \hat{\theta}_{n}\right)$, and $\left(\tilde{\theta}_{2}, \ldots, \tilde{\theta}_{n}\right)$ such that $u\left(f\left(\hat{\theta}_{1}, \tilde{\theta}_{2} \ldots, \tilde{\theta}_{n}\right), \hat{\theta}_{1}\right)>u\left(f\left(\hat{\theta}_{1}, \hat{\theta}_{2}, \ldots, \hat{\theta}_{n}\right), \hat{\theta}_{1}\right)$. (Note that we maintain Assumption A1.) Let deception $\gamma$ be defined as follows: $\gamma\left(\hat{\theta}_{1}, \hat{\theta}_{2}, \ldots, \hat{\theta}_{n}\right)=\left(\hat{\theta}_{1}, \tilde{\theta}_{2} \ldots, \tilde{\theta}_{n}\right)$ and $\gamma\left(\theta_{1}, \theta_{2}, \ldots, \theta_{n}\right)=\left(\theta_{1}, \theta_{2}, \ldots, \theta_{n}\right)$ for all $\left(\theta_{1}, \theta_{2}, \ldots, \theta_{n}\right) \neq\left(\hat{\theta}_{1}, \hat{\theta}_{2}, \ldots, \hat{\theta}_{n}\right)$. Note that $u_{1}(f \circ \gamma)>u_{1}(f)$ holds. Also, since $f$ is not weakly generation-efficient in the range, there exists $\delta \in \Delta$ such that $\sum_{j=1}^{i} u_{j}(f \circ \delta)>\sum_{j=1}^{i} u_{j}(f)$ for all $i \in N$. We initially assume that $\delta$ is a deterministic deception and we discuss the case of random deception at the end.

Following Smith (1992), we refer to $T$ periods from $z T$ to $(z+1) T-1$ for any $z \in \mathbb{Z}$ as an overlap. We divide each overlap into two parts: the first $Q$ periods and the remaining $T-Q$ periods. During the first $Q$ periods, the agents who are alive during that overlap, will deceive according to $\delta$, while during the last $T-Q$ periods, the agents deceive according to $\gamma$, which rewards agent 1 . (Note that even though agent 1 gains ex ante from both deceptions, he can be worse off for some realized profile of types when the agents deceive according to $\delta$.) Thus, let $\theta^{t}=\left(\theta_{1}^{t}, \ldots, \theta_{n}^{t}\right)$ be the profile of types in period $t$. Then, the period $t$ deception is: $\pi\left(\theta^{t}\right)=\delta\left(\theta^{t}\right)$ if $t \bmod T<Q$ and $\pi\left(\theta^{t}\right)=\gamma\left(\theta^{t}\right)$ otherwise.

The histories of types and messages are defined similar to before: $\zeta_{0}=\theta^{0}$ and $\zeta_{t}=\left(\zeta_{t-1}, \theta^{t-1}\right)$ for $t>0$, and $\mu_{t}=\left(\mu_{t-1}, m^{t-1}\right)$ with $\mu_{0}=\emptyset$, where $m^{t}$ are period $t$ messages. ${ }^{22}$ The period $t$ history is $\left(\zeta_{t}, \mu_{t}\right)$.

We are now ready to define the strategies $s^{\prime}$, but first we provide a verbal description. The agents play according to $s$, but they deceive according to $\pi$. That is, if their true types are $\theta$, they act as if their types were $\pi(\theta)$. They will do so as long as no deviation has occurred. If there is ever a deviation, then the agents start conditioning $s$ on their true types, but they still behave as if the types that were drawn before the (first) deviation, were the ones given by the deception. Said differently, $s$ depends on the history of types. We identify the first period when a deviation has occurred. Then, when conditioning $s$ on the history of types, the types before the deviation are

\footnotetext{
${ }^{21}$ From the proof of Claim 1, it follows that to eliminate unanimous, but untruthful messages, it is enough if agent $z-1$ can trigger part $1 \mathrm{c}$ by challenging the types of agents $z-2$ and $z-1$. Contrast it with Footnote 20.

22 The types of agents $-n+1, \ldots,-1$ before period 0 are irrelevant and, therefore, omitted.
} 
obtained by applying $\pi$ to the true types, while the types after the deviation correspond to the true types.

We define $s^{\prime}$ recursively with the help of a couple of auxiliary variables. Let $\zeta_{0}^{\prime}=\pi\left(\theta^{0}\right)$ and $d\left(\zeta_{0}, \mu_{0}\right)=1$. The period 0 strategies are $s^{\prime}\left(\zeta_{0}, \mu_{0}\right)=s\left(\zeta_{0}^{\prime}, \mu_{0}\right)$. Also, let $d\left(\zeta_{1}, \mu_{1}\right)=d\left(\zeta_{0}, \mu_{0}\right) \cdot \mathbf{1}_{\left\{s^{\prime}\left(\zeta_{0}, \mu_{0}\right)=s\left(\zeta_{0}^{\prime}, \mu_{0}\right)\right\}}$, where $\mathbf{1}_{\{E\}}$ is an indicator function taking value 1 if $E$ is true and 0 otherwise. In period 1 , if $d\left(\zeta_{1}, \mu_{1}\right)=1$, then $\zeta_{1}^{\prime}=\left(\zeta_{0}^{\prime}, \pi\left(\theta^{1}\right)\right)$; otherwise, $\quad \zeta_{1}^{\prime}=\left(\zeta_{0}^{\prime}, \theta^{1}\right)$ Let $\quad s^{\prime}\left(\zeta_{1}, \mu_{1}\right)=s\left(\zeta_{1}^{\prime}, \mu_{1}\right) \quad$ and $d\left(\zeta_{2}, \mu_{2}\right)=d\left(\zeta_{1}, \mu_{1}\right) \cdot \mathbf{1}_{\left\{s^{\prime}\left(\zeta_{1}, \mu_{1}\right)=s\left(\zeta_{1}^{\prime}, \mu_{1}\right)\right\}}$. Suppose we have defined the variables up to period $t-1$. In period $t$, if $d\left(\zeta_{t}, \mu_{t}\right)=1$, then $\zeta_{t}^{\prime}=\left(\zeta_{t-1}^{\prime}, \pi\left(\theta^{t}\right)\right)$; otherwise, $\zeta_{t}^{\prime}=\left(\zeta_{t-1}^{\prime}, \theta^{t}\right)$. Let $s^{\prime}\left(\zeta_{t}, \mu_{t}\right)=s\left(\zeta_{t}^{\prime}, \mu_{t}\right)$ and $d\left(\zeta_{t+1}, \mu_{t+1}\right)=d\left(\zeta_{t}, \mu_{t}\right) \cdot \mathbf{1}_{\left\{s^{\prime}\left(\zeta_{t}, \mu_{t}\right)=s\left(\zeta_{t}^{\prime}, \mu_{t}\right)\right\}}$.

We make a couple of observations. First, suppose we are in period $t$ and a deviation from $s^{\prime}$ has occurred in one of the previous periods, that is, $d\left(\zeta_{t}, \mu_{t}\right)=0$. Then, since past types do not affect current or future payoffs, by construction, $s^{\prime}$ imply Nash equilibrium play from that point on. Thus, we only need to verify whether nobody has incentives to deviate from $s^{\prime}$ on the equilibrium path, that is, when $d\left(\zeta_{t}, \mu_{t}\right)=1$ for some $t$. Second, suppose we are in period $t$, no deviation from $s^{\prime}$ has occurred so far, and the history is $\left(\zeta_{t}, \mu_{t}\right)$. Suppose an agent is contemplating a deviation. Then, clearly, the period $t$ outcomes and the continuation payoffs from period $t+1$ onwards that this agent can induce by deviating are exactly the same that he can induce by deviating from $s$ after history $\left(\zeta_{t}^{\prime}, \mu_{t}\right)$.

In the remaining analysis, all that matters is which period of the overlap we consider. Therefore, $t$ will now only take values in $\{1, \ldots, T\}$. Thus, agent $n$ is born at the beginning of period 1 , while agent 1 dies at the end of period $T$. Occasionally, it will be convenient to refer to agent $i+1$ in period $T$ as agent $i$ in period 0 .

If agent $i \in N$ deviates in period $t \in\{1, \ldots, T\}$, then besides the period $t$ payoff, he also expects a continuation payoff from the next period onwards. For the comparison purposes, it will be convenient to write this continuation payoff as $(i T-t) v$. That is, after the deviation, agent $i$ will still live for $(i T-t)$ periods. Hence, $v$ is the average, ex ante, per-period payoff during these $(i T-t)$ periods.

We need to prove that nobody wants to deviate from $s^{\prime}$ on the equilibrium path. We will first show that agent 1 does not want to deviate during periods $Q+1, \ldots, T$. Next, we will show that he also does not have incentives to deviate in periods $1, \ldots, Q$ when $T-Q$ is sufficiently large. Finally, we will show that any agent $i>1$ also does not want to deviate in periods $1, \ldots, T$ for sufficiently large $Q$.

Claim 2 Agent 1 has no incentives to deviate during periods $Q+1, \ldots, T$.

Proof of Claim 2 The proof is by contradiction. Suppose there is $t \in\{Q+1, \ldots, T\}$ and a history with period $t$ state $\left(\theta_{1}, \theta_{2}, \ldots, \theta_{n}\right)$ when agent 1 has incentives to deviate. Note that during periods $Q+1, \ldots, T$, the agents deceive according to $\gamma$. Thus, there exist an alternative $a$ and a continuation payoff $(T-t) v$ that agent 1 can obtain by deviating, such that the following inequality holds:

$$
u\left(f \circ \gamma\left(\theta_{1}, \theta_{2}, \ldots, \theta_{n}\right), \theta_{1}\right)+(T-t) u_{1}(f \circ \gamma)<u\left(a, \theta_{1}\right)+(T-t) v .
$$


The above inequality is still true if we replace $u_{1}(f \circ \gamma)$ with $u_{1}(f)$. But as noted before, we can find a history when the agents follow strategies $s$ such that the true period $t$ state is $\gamma\left(\theta_{1}, \theta_{2}, \ldots, \theta_{n}\right)$ and agent 1 can obtain the same $a$ and $(T-t) v$ by deviating from $s$. Since the agents do not lie about the type of agent 1 under $\gamma$, it follows then from the above inequality (with $u_{1}(f \circ \gamma)$ replaced by $u_{1}(f)$ ) that agent 1 also has a profitable deviation from $s$, which contradicts the assumption that $s$ is an SPE.

To prove the following two claims, our approach will be as follows. When considering a deviation by some agent in some period, we will lower-bound the payoff that he expects from following the strategy, and we will upper-bound the payoff that he expects from deviating from that strategy, and we will show that the former payoff is higher than the latter for sufficiently large $T$. When we lower-bound agent's payoff, we can assume that his current period payoff is $\underline{u}=\inf _{(a, \theta) \in A \times \Theta} u(a, \theta)$, irrespective of the actual state of the world. Similarly, when we upper-bound agent's payoff, we can assume that his current period payoff is $\bar{u}=\sup _{(a, \theta) \in A \times \Theta} u(a, \theta)$, again irrespective of the actual state of the world. For this reason, we can ignore what is the actual state of the world, when analysing the incentives to deviate.

Also, for the following claims, we need an upper-bound on the continuation payoff $(i T-t) v$ that agent $i$ can obtain by deviating in period $t$. We use the fact that $s$ is an SPE. Thus, suppose agent $i$ deviates from $s$ in period $t$ and state $\left(\theta_{1}, \ldots, \theta_{n}\right)$ and, as a result, period $t$ outcome is alternative $a$ and this agent expects $(i T-t) v$ in the continuation. Since $s$ is SPE, this deviation cannot be profitable:

$$
u\left(f\left(\theta_{1}, \ldots, \theta_{n}\right), \theta_{i}\right)+T \sum_{j=1}^{i} u_{j}(f)-t u_{i}(f) \geq u\left(a, \theta_{i}\right)+(i T-t) v .
$$

Since we want to upper-bound $(i T-t) v$, we set $u\left(f\left(\theta_{1}, \ldots, \theta_{n}\right), \theta_{i}\right)=\bar{u}$ and $u\left(a, \theta_{i}\right)=\underline{u}$. Hence, the upper-bound on the continuation payoff from any deviation, in any state, for any $i$ and $t$ is given by

$$
(i T-t) v \leq \bar{u}-\underline{u}+T \sum_{j=1}^{i} u_{j}(f)-t u_{i}(f) .
$$

We will use this expression in the continuation.

Claim 3 For sufficiently large $T-Q$, agent 1 has no incentives to deviate during periods $1, \ldots, Q$.

Proof of Claim 3 Since $u_{1}(f \circ \delta)>u_{1}(f)$ holds, it is easy to see that if agent 1 does not have incentives to deviate in period $Q$, then he also has no incentives to deviate in periods $t \in\{1, \ldots, Q-1\}$. Hence, we focus on period $t=Q$. His worst payoff from sticking to the deception, whatever is the state of the world, is

$$
\underline{u}+(T-Q) u_{1}(f \circ \gamma)
$$


while his payoff from any deviation can be upper-bounded by

$$
\bar{u}+(T-Q) v \leq 2 \bar{u}-\underline{u}+(T-Q) u_{1}(f),
$$

where we have used (1). Thus, agent 1 has no incentives to deviate in period $Q$ if

$$
\underline{u}+(T-Q) u_{1}(f \circ \gamma) \geq 2 \bar{u}-\underline{u}+(T-Q) u_{1}(f) .
$$

Since $u_{1}(f \circ \gamma)>u_{1}(f)$, we can find $T-Q$ such that the above inequality is indeed satisfied.

Claim 4 For sufficiently large $Q$, no agent $i>1$ has incentives to deviate during periods $1, \ldots, T$.

Proof of Claim 4 Consider any agent $i>1$. Agent $i$ 's decision in which period to deviate, depends on the relative magnitudes of $u_{i}(f \circ \delta), u_{i}(f \circ \gamma)$, and $u_{i}(f)$. Consider first the periods $t=1, \ldots, Q$. If $u_{i}(f \circ \delta)>u_{i}(f)$, then the agent will want to benefit from the deception $\delta$ as long as possible and if he decides to deviate, then it will be in period $Q$. (More precisely, if the agent has incentives to deviate for any $t \in\{1, \ldots, Q\}$, then he necessarily has incentives to deviate in period $Q$.) If $u_{i}(f \circ \delta)<u_{i}(f)$, then the agent will want to deviate as soon as possible. It is convenient to set the period when the agent deviates as period 0 , which is equivalent to agent $i+1$ deviating in period $T$. Now, consider the periods $t=Q+1, \ldots, T$. If $u_{i}(f \circ \gamma)>u_{i}(f)$, then the agent will want to benefit from the deception $\gamma$ as long as possible and if he decides to deviate, then it will be in period $T$ or, equivalently, period 0 for agent $i-1$. If $u_{i}(f \circ \gamma)<u_{i}(f)$, then the agent will want to deviate as soon as possible, that is, in period $Q$. To sum up, we only need to consider two periods when an agent $i$ decides to deviate: periods 0 and $Q$.

Consider $t=0$. The worst payoff to agent $i$ from sticking to the deception, irrespective of the state of the world, is

$$
\underline{u}+Q \sum_{j=1}^{i} u_{j}(f \circ \delta)+(T-Q) \sum_{j=1}^{i} u_{j}(f \circ \gamma),
$$

while his payoff from deviating can be upper-bounded by

$$
\bar{u}+i T v \leq 2 \bar{u}-\underline{u}+Q \sum_{j=1}^{i} u_{j}(f)+(T-Q) \sum_{j=1}^{i} u_{j}(f)
$$

where we have again used (1). Since $\sum_{j=1}^{i} u_{j}(f \circ \delta)>\sum_{j=1}^{i} u_{j}(f)$, then for a fixed $T-Q$, we can always find $Q$ such that the payoff from following the deception exceeds the one from the deviation. The case for $t=Q$ is similar and, therefore, omitted.

To summarize, we can always find $T-Q$ and $Q$ and, hence, $T$ large enough, so that no agent has incentives to deviate from the deception $\delta$ for the first $Q$ periods, 
followed by the deception $\gamma$ for the remaining $T-Q$ periods in each overlap. That is, no agent has incentives to deviate from $s^{\prime}$. Hence, $f$ is not implemented.

Finally, if $\delta$ is a random deception, then we can always construct a sequence of deterministic deceptions that on average give almost the same payoff as $\delta$. More precisely, if $\sum_{j=1}^{i} u_{j}(f \circ \delta)>\sum_{j=1}^{i} u_{j}(f)$ for all $i \in N$ and some $\delta \in \Delta$, then there exist $\delta_{1}, \ldots, \delta_{R}$ for some $R$ such that $\delta_{l} \in D$ for all $l=1, \ldots, R$ and $\sum_{l=1}^{R} \sum_{j=1}^{i} u_{j}\left(f \circ \delta_{l}\right)>R \sum_{j=1}^{i} u_{j}(f)$ for all $i \in N$. Arguments similar to the above ones can be used to show that we can always find $T-Q R$ and $Q$ and, hence, $T$ large enough, so that no agent has incentives to deviate when in each overlap, the agents first deceive $Q$ times according to $\delta_{1}, \ldots, \delta_{R}$ for $Q R$ periods in total and then according to $\gamma$ for the remaining $T-Q R$ periods.

\section{References}

Abreu D, Sen A (1990) Subgame perfect implementation: a necessary and almost sufficient condition. J Econ Theory 50(2):285-299

Azacis H, Vida P (2019) Repeated implementation: a practical characterization. J Econ Theory 180:336-367

Chambers CP (2004) Virtual repeated implementation. Econ Lett 83(2):263-268

Cremer J (1986) Cooperation in ongoing organizations. Q J Econ 101(1):33-49

Hayashi T, Lombardi M (2019) One-step-ahead implementation. J Math Econ 83:110-126

Kalai E, Ledyard JO (1998) Repeated implementation. J Econ Theory 83(2):308-317

Kandori M (1992) Repeated games played by overlapping generations of players. Rev Econ Stud 59(1):81-92

Kreps DM, Wilson R (1982) Sequential equilibria. Econometrica 50(4):863-894

Lee J, Sabourian H (2011) Efficient repeated implementation. Econometrica 79(6):1967-1994

Mezzetti C, Renou L (2017) Repeated Nash implementation. Theor Econ 12(1):249-285

Moore J, Repullo R (1988) Subgame perfect implementation. Econometrica 56(5):1191-1220

Salant DJ (1991) A repeated game with finitely lived overlapping generations of players. Games Econ Behav 3(2):244-259

Shinotsuka T, Suga K, Suzumura K, Tadenuma K (2007) Equity and efficiency in overlapping generations economies. In: Roemer J, Suzumura K (eds) Intergenerational equity and sustainability. Palgrave Macmillan, London, pp 20-35

Smith L (1992) Folk theorems in overlapping generations games. Games Econ Behav 4(3):426-449

Vartiainen H (2007) Subgame perfect implementation: a full characterization. J Econ Theory 133(1):111-126

Publisher's Note Springer Nature remains neutral with regard to jurisdictional claims in published maps and institutional affiliations. 